\title{
POCUS in perioperative medicine: a North American perspective
}

\author{
Lorenzo De Marchi ${ }^{1}$ and Massimiliano Meineri ${ }^{2^{*}}$
}

\begin{abstract}
Ultrasound (US) performed at the point of care has found fertile ground in perioperative medicine. In the hands of anesthesiologists, transesophageal echocardiography (TEE) has become established as a powerful diagnostic and monitoring tool in the perioperative care of cardiac and non-cardiac patients. A number of point-of-care US (POCUS) applications are relevant to perioperative care, including airway, cardiac, lung and gastric US. Although guidelines exist to define the scope of practice for basic and advanced TEE, there remains a lack of such guidelines for perioperative point-of-care ultrasound (POCUS), despite a number of recent calls for action in the academic anesthesia community. POCUS training has been integrated into anesthesia residency curricula in Canada and the United States of America (USA). However, a nation-wide curriculum is still lacking. Many limitations to the development of perioperative POCUS curricula exist, including the need to define the scope of practice and design integrated longitudinal learning approaches. The main anesthesiologist societies in both the USA and Canada are promoting the development of guidelines and have introduced POCUS courses into their national conferences. Although bedside US imaging has been integrated into the curricula of many medical schools in North America, the need for specific national guidelines for the training and practice of POCUS in the perioperative setting by anesthesiologists is crucial to the further development of POCUS in perioperative medicine.
\end{abstract}

Keywords: Point-of-care ultrasound, Perioperative medicine, Guidelines, Hand-held ultrasound devices

\section{Introduction}

Point-of-care ultrasound (POCUS) refers to the use of ultrasound (US) examinations at a patient's bedside by a primary healthcare provider to answer a limited number of specific diagnostic questions, guide treatment and invasive procedures. In the past decade, the role of this technology during the perioperative period has become increasingly recognized.

The use of US has become an established standard of care in most operating rooms for several applications, such as regional anesthesia and central line insertion [1]. Concurrently, transesophageal echocardiography (TEE) performed by anesthesiologists has become an established practice in the cardiac operating room, supported by specific guidelines in both the United States

\footnotetext{
*Correspondence: Massimiliano.Meineri@uhn.ca

${ }^{2}$ Department of Anesthesia and Pain Management, Toronto General Hospital, University Health Network, University of Toronto, 200 Elizabeth Street EN 3-400, Toronto, ON M5G 2C4, Canada

Full list of author information is available at the end of the article
}

of America (USA) and Canada [2, 3]. This has helped increase the availability of US systems in the operating room and develop specific postgraduate curricula to teach US-guided line insertion and confirmation of placement and regional blocks [4-6]. Additionally, handheld systems have become increasingly available for many physicians and acute care teams $[7,8]$.

A number of specific applications of POCUS assist providers in responding to the challenges of perioperative anesthetic care. Focused cardiac US [9] has been used in the perioperative assessment of elective [10] and urgent [11] surgical cases and has significantly impacted patient management [12-14]. POCUS may also play key roles in the management of hemodynamic instability and cardiac arrest [15]. Lung US not only exhibits high accuracy in the differential diagnosis of hypoxia [16] but also has been successfully used intraoperatively to detect tracheal intubation [17], exclude main stem bronchial intubation and confirm lung isolation in thoracic surgery [18, 19]. Upper airway US has been demonstrated to be more 
reliable than manual palpation in identifying the cricothyroid membrane [20] potentially reducing the risk of complications or failure in case of an emergency surgical airway.

Finally, while controversial, gastric US has become a unique tool in the hands of anesthesiologists for quantifying gastric contents and more objectively assessing aspiration risk [21]. However, this method is user dependent and subjective and is not standard of care in deciding if the patient is properly NPO.

More recently, specific perioperative scanning protocols have been proposed [22], and extensive reviews have advocated the need for anesthesiologists to perform perioperative POCUS [23]. Editorials in major anesthesia journals in the USA [24-26] and Canada [27] have clearly indicated that POCUS is the tool of the future in perioperative medicine. In 2011, an editorial by Johnson advocated for the development of POCUS into a standard of care for anesthesiologists [28]. Despite this editorial and other call for action, little progress has been made, and there exists no clear track toward a unified POCUS certification for anesthesiologists in North America.

In a recent survey of members of the Society of Cardiovascular Anesthesiologists, among 349 cardiac anesthesiologists who responded, most of whom were practicing in the USA, fewer than half had integrated focused cardiac US into their daily practice, and only $40 \%$ felt comfortable teaching this technique [29].

Although comprehensive [30] guidelines for the practice of POCUS are well established for emergency medicine and critical care in both the USA [30] and Canada [31], they are not immediately transferable to perioperative care, given the different scopes of practice. Another potential limitation restricting increases in the frequency with which POCUS is utilized is the relatively small number of anesthesiologists practicing in critical care in North America.

\section{Where are we?}

The American Society of Echocardiography and the Society of Cardiovascular Anesthesiologists have established guidelines regarding basic perioperative TEE that define the use of TEE as a monitoring tool outside of the cardiac operating room. National Board of Echocardiography certification in basic perioperative TEE is available after the completion of a written exam (for Testamur status) and the submission of a complete case log (for Diplomate status). Basic TEE certification is not supported by updated Canadian guidelines and is limited to the intraoperative setting. Training in basic perioperative TEE nonetheless requires a significant time commitment and supervision.
Basic TEE is an ideal tool for perioperative hemodynamic monitoring, assessments of volume status, guidance for fluid administration, and rescue in cases involving hemodynamic instability [32]. The integration of basic TEE training into the residency curriculum [33] should be considered to offer the full potential of this tool to the next generation of providers [23, 34].

Similarity to focused TTE [9], a more simplified "focused" TEE limited to five views with the objective of answering dichotomous questions has been successfully used in emergency medicine [35] and critical care [36]. However, the intraoperative use of this technique has not been described to date.

The feasibility and effectiveness of basic focused TTE in Canadian anesthesia residency programs were described more than 5 years ago [4]. Research has demonstrated that in the USA, a comprehensive perioperative US curriculum significantly impacted anesthesia residents' clinical assessment skills [34]. However, focused cardiac US teaching was found to be uncommon in anesthesia residency programs in the USA [37, 38], and a recent survey indicated that only 8 of 17 anesthesia residency programs in Canada offered any form of POCUS training [38]. In contrast, over $90 \%$ of cardiovascular anesthesiologists surveyed in the USA responded that focused cardiac US should be integrated into every anesthesia residency curriculum [37].

\section{What is next?}

A structured pathway for developing postgraduate curricula has been proposed for critical care anesthesiologists [39] and for specific perioperative POCUS applications [40, 41].

POCUS curriculum development for anesthesia residency programs must be considered in the context of the new framework of competency by design [42]. The current Canadian model implies that all anesthesia training is built to be tested against all applicable CanMEDS roles. POCUS appears to be relevant to all seven of these roles (medical expert, professional, communicator, collaborator, leader, scholar and professional). From this perspective, POCUS would not be regarded as a separate technical skill but would instead be integrated into daily clinical practice. This development would require a number of steps, including defining the scope of practice for perioperative POCUS and designing a longitudinal curriculum that accounts for current evidence regarding training duration and structure $[4,43]$, simulator use, number of scans necessary to achieve proficiency, $[9,44-$ 46] assessments of competence [47] and skill retention $[48,49]$. National or international expert consensus may be required in areas for which no supporting evidence is available. 
A separate challenge is to train the trainers; this challenge will lead to the creation of a certification pathway. Definitions of learning requirements will have to consider prior US experience hands-on learning and portfolio building will be limited by access to supervision and a lack of dedicated scanning time [50].

The introduction of integrated US teaching into medical school curricula is becoming standard across North America, and current graduates are therefore being trained to integrate US into clinical decision making. Residency curricula have a mandate to build on this foundation, and practicing anesthesiologists will need to prepare themselves for new teaching challenges.

Anesthesiologists are the main players in the perioperative care of surgical patients, from preoperative assessment to post-operative pain management. Therefore, they are the natural leaders in the context of the newly proposed perioperative surgical home model. With this prospect of more comprehensive longitudinal patient management, POCUS will find a fertile field of applications, given its proven roles in facilitating problem solving [51] and ensuring patient safety [34] during the pre-, intra- and post-operative periods.

POCUS requires adequate privileges, credentialing and oversight for quality assurance. At present, privileges to perform POCUS are currently granted by individual departments. There is no exemption for POCUS; this status may be problematic for medico-legal and remuneration purposes. In Canada and the USA, local regulations require electronic image storage and written reports for billing purposes. The availability of pictures archiving and communication systems (PACS) or web-based storage systems represents a significant concern, especially for community hospitals. Given the low remuneration for POCUS exams in both Canada and the USA, it is difficult to build a business model to support the practice of these examinations and justify the setup and maintenance costs of an image storage infrastructure. However, this challenge is a major limitation with respect to quality assurance and control.

\section{The future}

An increasing number of anesthesia residency programs are integrating a mandatory POCUS curriculum. This development is echoed by increased interest in POCUS training targeted to practicing anesthesiologists. Nonetheless, guidelines are lacking.

As a start, in 2016, the Canadian Anesthesiologist's Society formed a task force to develop a perioperative POCUS consensus statement that reflected the views of POCUS experts in all academic centers across the country. The first nation-wide, full-day perioperative POCUS course was held in Vancouver in June 2016. This course addressed all perioperative POCUS applications identified by the Perioperative POCUS Consensus Group.

The Canadian Emergency Ultrasound Society (CEUS) also developed the Emergency Department Echo Course in 2001, which became the standard POCUS certification course for emergency physicians in Canada. CEUS rebranded itself as the Canadian Point of Care Ultrasound Society (CPoCUS) (https://www.cpocus.ca) in 2016, and based on the strength of its certifying platform, offers certifications to anesthesiologists by combining different modules to fit applications of interest during the perioperative period.

In contrast, no major anesthesia society in the USA has embarked on the task of defining the scope of practice for POCUS or a path towards POCUS certification.

A special POCUS interest group was established last year within the American Society of Regional Anesthesia (ASRA).

However, the 2-day workshop on POCUS in anesthesia organized for 2017 [52] specially focused on focus assessed transthoracic echocardiography (FATE) certification and did not include all of the aforementioned components.

Thus far, the only other attempt to create a comprehensive POCUS certification has been offered by the Alliance for Physician Certification \& Advancement (APCA) [53], a physician-centric council spun out of the American Registry for Diagnostic Medical Sonography (ARDMS).

The APCA is currently working to provide, a POCUS Fundamental Certificate covering basic knowledge regarding US. This certificate will be followed by a series of POCUS Clinical Certificates (cardiac, GI, lung, abdominal trauma, and GU certificates, among others) [53].

The acquisition of a predetermined number of POCUS Clinical Certificates will earn the provider a certificate in POCUS specific to a determined specialty.

The first available certification will be in emergency medicine. Additional specialty-dedicated certifications, including a certification in anesthesia, are planned for development in the future.

Even if the APCA certification system does not address the extension of training necessary to reach competency in POCUS, it may nonetheless represent a useful practical tool to evaluate the results of an appropriately formulated learning curriculum. Although a minimum number of scans will likely continue to be required, the creation of a curriculum based on a competency-based education model that will be more focused on the achievement of certain milestones than a predetermined number of exams will help to finally achieve this goal.

It is hoped that the increased interest in POCUS that has recently been expressed in numerous anesthesia 
publications will promote a movement that will lead to the formulation of a clear curriculum and guidelines by our professional societies.

To answer to the "call to action" from Mahmood et al. in last year's June issue of Anesthesia \& Analgesia [16], the present generation of anesthesiologists must have a well-defined and structured learning path to be able to teach subsequent generations.

\section{Abbreviations}

POCUS: point-of-care ultrasound; US: ultrasound; TEE: transesophageal echocardiography.

\section{Authors' contributions}

LDM helped create the manuscript outline, wrote part of the first draft and edited the subsequent version of the manuscript. MM conceived the original idea, helped create the manuscript outline, wrote part of the first draft and edited the subsequent version of the manuscript. All authors read and approved the final manuscript.

\section{Author details}

${ }^{1}$ Department of Anesthesia, Georgetown University, MedStar Georgetown University Hospital, 3800 Reservoir Road NW, CCC Building, Lower Level, Washington, DC, USA. ${ }^{2}$ Department of Anesthesia and Pain Management, Toronto General Hospital, University Health Network, University of Toronto, 200 Elizabeth Street EN 3-400, Toronto, ON M5G 2C4, Canada.

\section{Acknowledgements}

None.

\section{Competing interests}

The authors declare that they have no competing interests.

\section{Availability of data and materials}

No patient data are involved.

\section{Consent for publication}

Not applicable.

\section{Ethics approval and consent to participate}

Not applicable.

\section{Funding}

This manuscript was not supported by any funding.

\section{Publisher's Note}

Springer Nature remains neutral with regard to jurisdictional claims in published maps and institutional affiliations.

Received: 17 May 2017 Accepted: 26 September 2017

Published online: 09 October 2017

\section{References}

1. Troianos CA, Hartman GS, Glas KE et al (2012) Special articles: guidelines for performing ultrasound guided vascular cannulation: recommendations of the American Society of Echocardiography and the Society of Cardiovascular Anesthesiologists. Anesth Analg 114(1):46-72

2. Beique F, Ali M, Hynes M et al (2006) Canadian guidelines for training in adult perioperative transesophageal echocardiography. Recommendations of the Cardiovascular Section of the Canadian Anesthesiologists' Society and the Canadian Society of Echocardiography. Can J Anaesth 53(10):1044-1060
3. Hahn RT, Abraham T, Adams MS et al (2013) Guidelines for performing a comprehensive transesophageal echocardiographic examination: recommendations from the American Society of Echocardiography and the Society of Cardiovascular Anesthesiologists. J Am Soc Echocardiogr 26(9):921-964

4. Tanzola RC, Walsh S, Hopman WM et al (2013) Brief report: focused transthoracic echocardiography training in a cohort of Canadian anesthesiology residents: a pilot study. Can J Anaesth 60(1):32-37

5. Arellano R, Nurmohamed A, Rumman A et al (2014) The utility of transthoracic echocardiography to confirm central line placement: an observational study. Can J Anaesth 61(4):340-346

6. Adler AC, Jablonka DH (2016) Bedside ultrasound: a tool for assessment of IV functionality. Anesthesiology 124(4):959

7. Seward JB, Douglas PS, Erbel R et al (2002) Hand-carried cardiac ultrasound (HCU) device: recommendations regarding new technology. A report from the Echocardiography Task Force on New Technology of the Nomenclature and Standards Committee of the American Society of Echocardiography. J Am Soc Echocardiogr 15(4):369-373

8. Beaulieu Y, Marik PE (2005) Bedside ultrasonography in the ICU: part 1. Chest 128(2):881-895

9. Spencer KT, Kimura BJ, Korcarz CE et al (2013) Focused cardiac ultrasound recommendations from the American Society of Echocardiography. J Am Soc Echocardiogr 26(6):567-581

10. Canty DJ, Royse CF, Kilpatrick D et al (2012) The impact on cardiac diagnosis and mortality of focused transthoracic echocardiography in hip fracture surgery patients with increased risk of cardiac disease: a retrospective cohort study. Anaesthesia 67(11):1202-1209

11. Botker MT, Vang ML, Grofte T et al (2014) Routine pre-operative focused ultrasonography by anesthesiologists in patients undergoing urgent surgical procedures. Acta Anaesthesiol Scand 58(7):807-814

12. Cowie $B$ (2011) Three years' experience of focused cardiovascular ultrasound in the peri-operative period. Anaesthesia 66(4):268-273

13. Markin NW, Gmelch BS, Griffee MJ et al (2015) A review of 364 perioperative rescue echocardiograms: findings of an anesthesiologist-staffed perioperative echocardiography service. J Cardiothorac Vasc Anesth 29(1):82-88

14. Adler AC (2017) Air embolism during cardiac catheterization and the role for anesthesia use of bedside ultrasound. Anesthesiology. PMID:28562374

15. Tsou PY, Kurbedin J, Chen YS et al (2017) Accuracy of point-of-care focused echocardiography in predicting outcome of resuscitation in cardiac arrest patients: a systematic review and meta-analysis. Resuscitation 114:92-99

16. Volpicelli G, Elbarbary M, Blaivas M et al (2012) International evidencebased recommendations for point-of-care lung ultrasound. Intensive Care Med 38(4):577-591

17. Das SK, Choupoo NS, Haldar R et al (2015) Transtracheal ultrasound for verification of endotracheal tube placement: a systematic review and meta-analysis. Can J Anaesth 62(4):413-423

18. Adler AC (2017) Perioperative point of care ultrasound in pediatric anesthesiology; a case series highlighting intra-operative diagnosis of hemodynamic instability and alteration of management. J Cardiothorac Vasc Anesth. PMID:28911897

19. Díaz-Gómez J, Ripoll JG, Ratzlaff RA, Tavazzi G, Via G, Mookadam F, Ramakrishna H, FASE F (2017) Perioperative lung ultrasound for the cardiothoracic anesthesiologist: emerging importance and clinical applications. J Cardiothorac Vasc Anesth. 9:10

20. You-Ten KE, Desai D, Postonogova T et al (2015) Accuracy of conventional digital palpation and ultrasound of the cricothyroid membrane in obese women in labour. Anaesthesia 70(11):1230-1234

21. Perlas A, Mitsakakis N, Liu L et al (2013) Validation of a mathematical model for ultrasound assessment of gastric volume by gastroscopic examination. Anesth Analg 116(2):357-363

22. Adler AC, Greeley WJ, Conlin F et al (2016) Perioperative Anesthesiology UltraSonographic Evaluation (PAUSE): a guided approach to perioperative bedside ultrasound. J Cardiothorac Vasc Anesth 30(2):521-529

23. Mahmood F, Matyal R, Skubas N et al (2016) Perioperative ultrasound training in anesthesiology: a call to action. Anesth Analg 122(6):1794-1804

24. Janelle GM, London MJ (2016) Perioperative ultrasound: the future is now. Anesth Analg 122(6):1734-1736 
25. Skubas NJ (2015) Teaching whole body point-of-care ultrasound: advancing the skills of tomorrow's anesthesiologists. Anesthesiology 123(3):499-500

26. Ramsingh D, Gudzenko V, Martin RD (2017) Point-of-Care Ultrasound: novel Technology to Routine Perioperative Assessment Tool. Anesth Analg 124(3):709-711

27. Denault A, Fayad A, Chen R (2013) Focused ultrasound is the next step in perioperative care. Can J Anaesth 60(8):741-747

28. Johnson DW, Oren-Grinberg A (2011) Perioperative point-of-care ultrasonography: the past and the future are in anesthesiologists' hands. Anesthesiology 115(3):460-462

29. Conlin F, Connelly NR, Eaton MP, et al. (2017) Perioperative use of focused transthoracic cardiac ultrasound: a survey of current practice and opinion. Anesth Analg. PMID:28537977

30. Marik PE, Mayo P (2008) Certification and training in critical care ultrasound. Intensive Care Med 34(2):215-217

31. Arntfield R, Millington S, Ainsworth C et al (2014) Canadian recommendations for critical care ultrasound training and competency. Can Respir J 21(6):341-345

32. American Society of Anesthesiologists CoE-PSSoTECPDA, 2015 FOR BOD/ HOD information. Statement on transesophageal echocardiography (Approved by the ASA House of Delegates on October 17, 2001, and last amended on October 28, 2015) 2015

33. Smith WB, Robinson AR 3rd, Janelle GM (2015) Expanding role of perioperative transesophageal echocardiography in the general anesthesia practice and residency training in the USA. Curr Opin Anaesthesiol 28(1):95-100

34. Ramsingh D, Rinehart J, Kain Z et al (2015) Impact assessment of perioperative point-of-care ultrasound training on anesthesiology residents. Anesthesiology 123(3):670-682

35. Arntfield R, Pace J, Hewak M, et al. (2015) Focused Transesophageal Echocardiography by Emergency Physicians is Feasible and Clinically Influential: Observational Results from a Novel Ultrasound Program. J Emerg Med 50(2):286-94. PMID:26508495

36. Vieillard-Baron A, Slama M, Mayo P et al (2013) A pilot study on safety and clinical utility of a single-use 72-h indwelling transesophageal echocardiography probe. Intensive Care Med 39(4):629-635

37. Conlin F, Roy Connelly N, Raghunathan Ket al (2016) Focused transthoracic cardiac ultrasound: a survey of training practices. J Cardiothorac Vasc Anesth 30(1):102-106

38. Mizubuti G, Allard R, Ho AM et al (2017) A survey of focused cardiac ultrasonography training in Canadian anesthesiology residency programs. Can J Anaesth 64(4):441-442

39. Fagley RE, Haney MF, Beraud AS et al (2015) Critical care basic ultrasound learning goals for American Anesthesiology Critical Care
Trainees: recommendations from an Expert Group. Anesth Analg 120(5):1041-1053

40. Perlas A, Van de Putte P, Van Houwe P et al (2016) I-AIM framework for point-of-care gastric ultrasound. Br J Anaesth 116(1):7-11

41. Via G, Hussain A, Wells M et al (2014) International evidence-based recommendations for focused cardiac ultrasound. J Am Soc Echocardiogr 27(7):683 e1-683 e33

42. Fraser AB, Stodel EJ, Jee R et al (2016) Preparing anesthesiology faculty for competency-based medical education. Can J Anaesth 63(12):1364-1373

43. Poelaert J, Mayo P (2007) Education and evaluation of knowledge and skills in echocardiography: how should we organize? Intensive Care Med 33(10):1684-1686

44. Millington SJ, Hewak M, Arntfield RT et al (2017) Outcomes from extensive training in critical care echocardiography: identifying the optimal number of practice studies required to achieve competency. J Crit Care 40:99-102

45. Arzola C, Carvalho JC, Cubillos J et al (2013) Anesthesiologists' learning curves for bedside qualitative ultrasound assessment of gastric content: a cohort study. Can J Anaesth 60(8):771-779

46. Gaspar HA, Morhy SS, Lianza AC et al (2014) Focused cardiac ultrasound: a training course for pediatric intensivists and emergency physicians. BMC Med Educ 14:25

47. Millington SJ, Arntfield RT, Hewak M et al (2016) The Rapid Assessment of Competency in Echocardiography Scale: validation of a Tool for Pointof-Care Ultrasound. J Ultrasound Med Off J Am Inst Ultrasound Med 35(7):1457-1463

48. Kimura BJ, Sliman SM, Waalen J et al (2016) Retention of Ultrasound Skills and Training in "Point-of-Care" Cardiac Ultrasound. J Am Soc Echocardiogr 29(10):992-997

49. Gaspar HA, Brunow de Carvalho W, Delgado AF (2016) How to train and maintain pediatric intensivists updated in focused cardiac ultrasound? Pediatr Crit Care Med 17(10):1015

50. Patrawalla P, Eisen LA, Shiloh A et al (2015) Development and validation of an assessment tool for competency in critical care ultrasound. J Grad Med Educ 7(4):567-573

51. Vignon P (2012) PRO: physician-performed ultrasound: the time has come for routine use in acute care medicine. Anesth Analg 115(5):999-1003

52. Perioperative Point-of-Care Ultrasound SIG ASoRA. Introduction to Perioperative Point-of-Care Ultrasound. February 25-26, 2017. https://www. asra.com/page/189/perioperative-point-of-care-ultrasound-sig

53. APCA AfPCaA-. Point-of-Care Ultrasound (POCUS) Academy. https://www apca.org/pocus\#! prerequisites-specialtycertifications

\section{Submit your manuscript to a SpringerOpen ${ }^{\odot}$ journal and benefit from:}

- Convenient online submission

- Rigorous peer review

- Open access: articles freely available online

- High visibility within the field

- Retaining the copyright to your article

Submit your next manuscript at springeropen.com 